\title{
Cross-cultural adaptation and validation of the Impact of Fixed Appliances Measure questionnaire in Brazil
}

\begin{abstract}
Ana Paula REBOUÇAS(a)
Cristiane Baccin BENDO(a)

Lucas Guimarães ABREU(a)

Elizabeth Maria Bastos LAGES(a)

Carlos FLORES-MIR ${ }^{(b)}$

Saul Martins PAIVA(a)
\end{abstract}

(a) Universidade Federal de Minas Gerais UFMG, Faculty of Dentistry, Department of Pediatric Dentistry and Orthodontics, Belo Horizonte, MG, Brazil.

(b) University of Alberta, Faculty of Medicine and Dentistry, Department of Dentistry, Edmonton, Canada.

Declaration of Interests: The authors certify that they have no commercial or associative interest that represents a conflict of interest in connection with the manuscript.

Corresponding Author:

Cristiane Baccin Bendo

crysbendo@yahoo.com.br

https://doi.org/10.1590/1807-3107bor- 2018.vol32.0014

Submitted: July 27, 2017

Accepted for publication: Nov 27, 2017

Last revision: Jan 23, 2018

\begin{abstract}
The aim of this study was to translate and cross-culturally adapt the condition-specific instrument Impact of Fixed Appliances Measure (IFAM), assessing its validity and reliability for use among Brazilian children/adolescents. The IFAM was translated, back-translated, cross-culturally adapted, and pilot-tested. The Brazilian version of the IFAM (B-IFAM) was tested on 161 10-to-18-year-old children/adolescents. Internal consistency was assessed using Cronbach's alpha $(\alpha)$ and test-retest reliability by intraclass correlation coefficients (ICC). Construct validity was conducted using Pearson correlation among the overall and subscales of the B-IFAM. Discriminant validity was evaluated by comparisons of B-IFAM's means and SD with children's/adolescents' sex (Student t-test). Internal consistency was 0.89 for overall score and $0.55-0.86$ for subscales. ICC for test-retest reliability was 0.81 for overall score and 0.55-0.78 for subscales. The overall score of the B-IFAM presented large correlation coefficients with most subscales $(r=0.52-0.74)$, supporting construct validity. Discriminant validity demonstrated statistically significant difference in the overall score, aesthetics, and physical impact subscales among female and male children's/adolescents' $(\mathrm{p}<0.05)$. In the conclusion, the B-IFAM overall score and some subscales demonstrated adequate psychometric properties regarding reliability and validity. The study achieved a specific-condition instrument feasible for use on Brazilian children/adolescents who wear fixed orthodontic appliances.
\end{abstract}

Keywords: Quality of Life; Surveys and Questionnaires; Orthodontics; Pediatric Dentistry

\section{Introduction}

In the last decades, there has been increasing interest in evaluation of the impact of health management outcomes on individuals' daily life and quality of life. The concept of oral health-related quality of life (OHRQoL) was developed to assess the effect of oral health management outcomes on people's lives and their overall quality of life. ${ }^{1}$ For this purpose, a number of instruments to be specifically used on children and adolescents and adults have been developed. The Child Oral Impact on Daily Performance (Child-OIDP) ${ }^{2}$ the Child Perceptions Questionnaire 
$(\mathrm{CPQ}),{ }^{3}$ the Early Childhood Oral Health Impact Scale (ECOHIS), ${ }^{4}$ and the Oral Health Impact Profile $(\mathrm{OHIP})^{5}$ are examples of OHRQoL instruments that have been extensively used in oral research targeted at this age range.

Many studies have evaluated the association between orthodontic treatment and OHRQoL using generic instruments. ${ }^{6,7,8}$ At the onset of treatment, OHRQoL often deteriorates because individuals are affected by pain, discomfort, and functional limitations. ${ }^{910}$ Following fixed appliances' debonding, however, quality of life is positively influenced as a result of a long-term improvement in emotional and social well-being. ${ }^{11}$ The understanding of this phenomenon allows orthodontists to inform their patients about the impact of fixed orthodontic appliances on their daily function shortly after bonding, during, and after orthodontic treatment. Such a strategy may likely contribute to reduce the negative impacts of fixed appliance therapy and, ultimately, to increase patient cooperation, and to avoid treatment dropouts. ${ }^{12}$

An instrument to measure OHRQoL can be classified as generic or condition-specific. The former evaluates the wider effects of different oral health management outcomes on quality of life, but may not present adequate responsiveness to identify changes resulting from a specific dental intervention. The latter, though, is more sensitive to specific clinical changes and may therefore be a valuable instrument to assess OHRQoL focused on one specific outcome, such as orthodontic treatment. ${ }^{13}$ To fill this gap in the literature, a specific measurement (Impact of Fixed Appliances Measure: IFAM) was developed and validated in United Kingdom to evaluate the impact of fixed orthodontic appliances on daily life of individuals between 10 and 18 years of age. ${ }^{14}$ Following cross-cultural adaptation, it has been also used in India. ${ }^{15}$ This measure has been considered a useful method to better understand the relationship between orthodontic treatment and OHRQoL, ${ }^{14}$ and could be a new instrument to be used on Brazilian patients. As the IFAM was developed in English, a validation process is required to use it in populations that use other languages on a daily basis. Thus, the aim of the present study was to translate and cross-culturally adapt a condition-specific instrument (IFAM) to evaluate the impact of fixed orthodontic appliances on daily life, and assess its validity and reliability for use among Brazilian 10-18-year-old children and adolescents.

\section{Methodology}

\section{Description of the Impact of Fixed Appliances Measure (IFAM)}

The IFAM is a condition-specific instrument developed to assess the impact of fixed orthodontic appliances on the daily life of children and adolescents between 10 and 18 years of age. This instrument is composed of 43 items distributed across nine subscales: aesthetics (A: five items; scores range from 5 to 25); functional limitation (FL: three items; scores range from 3 to 15); dietary impact (DI: six items; scores range from 6 to 30); oral hygiene impact (OHI: three items; scores range from 3 to 15); maintenance impact (MI: two items; scores range from 2 to 10); physical impact (PI: nine items; scores range from 9 to 45); social impact (SI: five items; scores range from 5 to 25): time constraints (TC: five items; scores range from 5 to 25); and travel/cost/inconvenience implications (TCII: five items; scores range from 5 to 25). Each item has five response options according to a Likert scale ranging from 1 to 5 , where $1=$ strongly disagree, 2 = disagree, 3 = neither agree nor disagree, $4=$ agree and $5=$ strongly agree. The overall score is computed by summing all the items in each subscale. The total ranges from 43 to 215 and a higher score denotes a greater negative impact of the fixed orthodontic appliances on the daily lives of children and adolescents. ${ }^{14}$

\section{Translation and cross-cultural adaptation of the IFAM}

The translation process and the cross-cultural adaptation of OHRQoL measures are based on standard guidelines that have been described by many authors in literature. ${ }^{16,17,18,19,20}$ For the IFAM, the following protocol was used. Initially, two translations (English to Brazilian Portuguese) were made independently by two bilingual translators. Both translators were native Brazilian Portuguese 
speakers, fluent in English and with significant experience in epidemiological studies. To determine the concept and equivalence of items, both translated versions were evaluated by an expert committee composed of three specialists in orthodontics and two specialists in pediatric dentistry, who were also native Brazilian Portuguese speakers fluent in English and with experience in oral research. Attention was given to the identification of possible shortcomings in the understanding of the instrument's items. Following discussion, the committee of specialists drafted a summarized version of the questionnaire in Brazilian Portuguese.

The summarized version of the questionnaire was backward translated into English by a native English translator who was blinded to the original instrument and not previously involved in the study. The back-translated version was sent to the authors of the original instrument, who provided some minor suggestions. The back-translator and the expert committee evaluated the back-translated version, taking into account the authors' comments and the original version.

This draft version in Brazilian Portuguese was then pilot-tested on a convenience sample of 15 individuals separated according to age group: five individuals between 10 and 12 years old, five individuals between 13 and 15 years old, and five individuals between 16 and 18 years old. This strategy allowed the researchers to evaluate a potential impact or different response based on all the target ages. The pilot test consisted of interviews conducted by one investigator. Participants were encouraged to explain the meaning of words and to provide synonyms and suggestions for words that might have been misunderstood. This method enabled the interviewer to evaluate the participants' understanding of the instructions, items, and response choices to detect any possible disagreement with the original version of the instrument. Following the pilot test, it was decided that no changes were needed. Therefore, this draft version was considered the final Brazilian Portuguese version of the IFAM instrument. The translation and the cross-cultural adaptation process are depicted in Figure.

\section{Assessment of validity and reliability of the Brazilian Portuguese version of the IFAM (B-IFAM)}

A convenience sample of 161 children and adolescents submitted to orthodontic treatment with fixed appliances and their parents/caregivers were selected from the orthodontic clinic at the Faculty of Dentistry of the Federal University of Minas Gerais (Belo Horizonte, Brazil) between 2015 and 2016. Inclusion criteria were children and adolescents between 10 and 18 years old who had been wearing metallic orthodontic fixed appliances for at least six months. Children and adolescents with dental traumatic injuries, cognitive disorders and craniofacial anomalies were excluded from the study. All individuals who met these criteria were invited to participate in the study.

Parents/caregivers completed a form with questions about the child/adolescent's age, parents/caregivers' level of education ( $\leq 8$ years of study and $>8$ years of study) and family income. Family income was assessed in terms of the Brazilian national monthly minimum wage (NMMW), which corresponded to US\$ 300.00 at the time of data collection. Family income was categorized as $\leq 2$ NMMWs and $>2$ NMMWs based on median value.

The B-IFAM is a self-assessment questionnaire. Children and adolescents were instructed to answer,

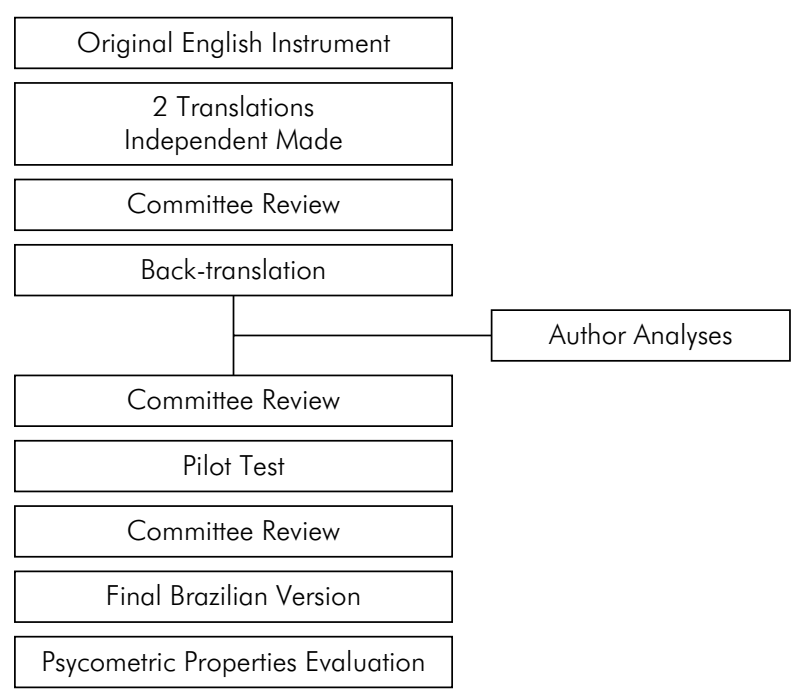

Figure. Flow chart of the translation and cross-cultural adaptation process. 
by their own, the B-IFAM in the university clinic waiting room before the appointment, except for the last two subscales, in which parents/caregiver were allowed to assist their sons/daughters. The original method of administration was maintained. The instrument was filled out twice by 141 individuals $(87.6 \%)$ for test-retest reliability. The second time was three weeks later, before orthodontic intervention. During these three weeks, they had not been submitted to any orthodontic intervention. If any question was raised, a researcher was available for clarification. The researcher ensured neutrality on participant's response.

\section{Ethical issues}

This study was approved by the Human Research Ethics Committee of the Federal University of Minas Gerais, Brazil (protocol number \#43747515.7.0000.5149). Participation was entirely voluntary, and an informed consent was signed by all children/adolescents and their parents/caregivers.

\section{Statistical analysis}

Data analyses were conducted using the Statistical Package for the Social Sciences (SPSS, version 22.0, IBM Corp., Armonk, NY, USA). Descriptive statistics included frequency distribution, ceiling and floor effects, means, and standard deviation (SD). Admissible floor or ceiling effects are less than or equal to $15 \% .{ }^{22}$ The internal consistency and test-retest reliability of the B-IFAM was determined. Internal consistency was estimated using Cronbach's alpha $(\alpha)$ coefficient. Values $\geq 0.70$ were considered acceptable. ${ }^{23}$ Testretest reliability was assessed using the intraclass correlation coefficient (ICC). The agreement level presented by the ICC was categorized as follows: $\leq 0.40$ weak correlation; $0.41-0.60$ moderate correlation; 0.61-0.80 good correlation; and 0.81-1.00 excellent correlation. ${ }^{24}$ Construct validity was tested using Pearson's correlation coefficient through association between the B-IFAM subscales' scores. Pearson product moment correlation coefficients are designated as small (0.10-0.29), medium (0.30-0.49), and large $(>0.50) .{ }^{25}$ The known-groups analysis, using the Student t test, established discriminant validity by comparisons of means and SD of the B-IFAM and sex.

\section{Results}

The study involved 161 children and adolescents with a mean age of $13.84(\mathrm{SD}=1.94)$. Sociodemographic characteristics and participants' orthodontic characteristics are presented in Table 1. No significant ceiling effect was observed, while floor effects appeared in the A, FL and SI subscales (Table 2).

\section{Reliability}

The B-IFAM presented a Cronbach's alpha coefficient of 0.89 for the overall score. For the subscales, the values ranged from 0.55 to 0.86 . Test-retest reliability assessed by ICC ranged between moderate agreement and excellent agreement. The ICC for overall score was 0.81 , indicating excellent agreement. Among the subscales, the ICC ranged from 0.55 to 0.78 , indicating moderate to good agreement (Table 2).

\section{Validity}

Table 3 demonstrates the intercorrelations between the overall and subscales' scores calculated by Pearson correlation to determine construct validity. The overall score of the B-IFAM presented large Pearson correlation coefficients, with most subscales ranging from 0.52 to 0.74 . Two subscales (MI and TCII) achieved

Table 1. Sociodemographic and orthodontic characteristics of the sample.

\begin{tabular}{lc}
\hline \multicolumn{2}{l}{ Variables } \\
\hline Children's/adolescents' age, mean (SD*) & $13.84(1.94)$ \\
Children's/adolescents' gender, n (\%) & $70(43.5)$ \\
$\quad$ Male & $91(56.5)$ \\
$\quad$ Female & \\
Family income, n (\%) & $101(62.7)$ \\
$\leq 2$ BMMW* & $60(37.3)$ \\
$>2$ BMMW* & \\
Parents'/caregivers' level of education, n (\%) & $62(32.5)$ \\
$\leq 8$ years of study & $99(61.5)$ \\
$>8$ years of study & \\
Angle's classification, n (\%) & $48(29.8)$ \\
$\quad$ Class I & $83(51.6)$ \\
Class II & $30(18.6)$ \\
\hline Class III & \\
\hline SD: standard deviation; BMMW: Brazilian Monthly Minimum Wage.
\end{tabular}


Table 2. Descriptive analysis and reliability of the B-IFAM to assess the impact of fixed appliances on daily life.

\begin{tabular}{lccccccc}
\hline Scale Impact of fixed appliance measure & Number of items & Mean & SD & Floor effects & Ceiling effects & Cronbach's alpha & ICC \\
\hline Overall score & 43 & 105.75 & 20.67 & 0 & 0 & 0.89 & 0.81 \\
Aesthetics & 5 & 9.77 & 3.95 & 16.8 & 0.6 & 0.82 & 0.60 \\
Functional limitation & 3 & 5.84 & 2.30 & 22.4 & 1.2 & 0.77 & 0.55 \\
Dietary impact & 6 & 15.88 & 5.48 & 6.2 & 1.2 & 0.81 & 0.64 \\
Oral hygiene impact & 3 & 10.25 & 3.20 & 4.3 & 11.2 & 0.86 & 0.62 \\
Maintenance impact & 2 & 5.77 & 2.10 & 10.6 & 3.7 & 0.55 & 0.68 \\
Physical impact & 9 & 23.32 & 6.35 & 2.5 & 0.6 & 0.81 & 0.78 \\
Social impact & 5 & 9.75 & 3.59 & 17.4 & 0.6 & 0.75 & 0.65 \\
Time constraints & 5 & 12.32 & 3.63 & 5.6 & 1.2 & 0.55 & 0.67 \\
Travel/cost/inconvenience implications & 5 & 12.94 & 4.12 & 3.7 & 1.2 & 0.74 & 0.58 \\
\hline
\end{tabular}

ICC: Intraclass correlation coefficients for test-retest.; ICCs $<0.40$ show poor to fair agreement, $0.41-0.60$ moderate agreement, $0.61-0.80$ good agreement and $>0.80$ excellent agreement.

medium correlation coefficients compared with overall B-IFAM. All coefficients were significant at 0.01 level.

For discriminant validity, correlation was carried out between female and male children/adolescents. Female children and adolescents presented higher scores compared with male for overall score $(p=0.006)$, A $(p=0.009)$ and PI $(p<0.001)$ (Table 4$)$.

\section{Discussion}

This study translated and cross-culturally adapted the IFAM to the Brazilian Portuguese language, and this alternative version was considered a reliable and valid instrument to be used with Brazilian children and adolescents. The method used to translate and cross-culturally adapt the instrument was carefully guided by standard recommendations described in literature. ${ }^{16,1718,19,20}$ The back-translated version resulted in a version very similar to the original questionnaire, therefore highlighting the suitability of the Brazilian Portuguese version achieved in the process. The participation of the instrument's author helped to increase the power of the final version in Brazilian Portuguese. ${ }^{19}$ The experience with the translation process and the orthodontic professionals involved in the expert committee helped to incorporate the best modifications to the Brazilian Portuguese language.

Floor effects appeared in the A, FL and SI subscales, which means that $16.8 \%, 22.4 \%$ and $17.4 \%$ respectively of the sample scored at the bottom of the scale. Therefore, no impact on aesthetics, functional limitations and social interactions were reported, perhaps as a result of adaptation to appliances, since children and adolescents in

Table 3. Construct validity: Pearson correlation of the Brazilian version of the IFAM

\begin{tabular}{|c|c|c|c|c|c|c|c|c|c|c|}
\hline Scale & OS & A & $\mathrm{FL}$ & DI & $\mathrm{OHI}$ & $\mathrm{Ml}$ & $\mathrm{Pl}$ & $\mathrm{SI}$ & $\mathrm{TC}$ & TCll \\
\hline OS & 1 & - & - & - & - & - & - & - & - & - \\
\hline A & $0.64^{* *}$ & 1 & - & - & - & - & - & - & - & - \\
\hline $\mathrm{FL}$ & $0.54^{* *}$ & $0.38^{* *}$ & 1 & - & - & - & - & - & - & - \\
\hline DI & $0.52^{* *}$ & 0.13 & 0.12 & 1 & - & - & - & - & - & - \\
\hline $\mathrm{OHI}$ & $0.57^{* *}$ & $0.32^{* *}$ & $0.35^{* *}$ & $0.20^{*}$ & 1 & - & - & - & - & - \\
\hline MI & $0.33^{* *}$ & 0.10 & 0.12 & $0.25^{* *}$ & 0.31 & 1 & - & - & - & - \\
\hline $\mathrm{PI}$ & $0.74^{* *}$ & $0.36^{* *}$ & $0.39^{* *}$ & $0.19^{*}$ & $0.43^{* *}$ & 0.11 & 1 & - & - & - \\
\hline SI & $0.71^{* *}$ & $0.68^{* *}$ & $0.30^{* *}$ & $0.20^{*}$ & $0.36^{* *}$ & 0.11 & $0.45^{* *}$ & 1 & - & - \\
\hline $\mathrm{TC}$ & $0.63^{* *}$ & $0.31^{* *}$ & $0.23^{* *}$ & $0.16^{*}$ & $0.26^{* *}$ & $0.17^{*}$ & $0.49^{* *}$ & $0.45^{* *}$ & 1 & - \\
\hline $\mathrm{TCl}$ & $0.46^{* *}$ & 0.11 & $0.24^{* *}$ & $0.20^{*}$ & 0.15 & $0.19^{*}$ & $0.17^{*}$ & $0.16^{*}$ & $0.25^{* *}$ & 1 \\
\hline
\end{tabular}

The impact of fixed orthodontic appliances measure: OS: Overall score; A: aesthetics; FL: functional limitation; DI: dietary impact; OHI: oral hygiene impact, MI: maintenance impact; PI: physical impact; SI: social impact; TC: time constraints; TCII: travel/cost/inconvenience implications. Pearson product moment correlation coefficients: small (0.10-0.29), medium (0.30-0.49), and large $(>0.50)^{25} ;{ }^{*} p<0.05 ;{ }^{* *} p<0.01$. 
Table 4. Discriminant validity: Comparison of the impact of fixed appliance measure with sex.

\begin{tabular}{lccc}
\hline \multirow{2}{*}{ Scale Impact of fixed appliance measure } & \multicolumn{3}{c}{ Sex } \\
\cline { 2 - 3 } & Female & Male & \\
Overall score & $109.63(19.4)$ & $100.71(21.3)$ & 0.006 \\
Aesthetics & $10.45(4.3)$ & $8.89(3.2)$ & 0.009 \\
Functional limitation & $6.03(2.1)$ & $5.59(2.5)$ & 0.222 \\
Dietary impact & $16.13(5.4)$ & $15.56(5.6)$ & 0.511 \\
Oral hygiene impact & $10.59(3.0)$ & $9.81(3.4)$ & 0.126 \\
Maintenance impact & $5.85(2.1)$ & $5.67(2.1)$ & 0.602 \\
Physical impact & $24.78(5.7)$ & $21.43(6.7)$ & $<0.001$ \\
Social impact & $10.13(3.6)$ & $9.24(3.5)$ & 0.119 \\
Time constraints & $12.42(3.5)$ & $12.19(3.9)$ & 0.689 \\
Travel/cost/inconvenience implication & $13.24(4.2)$ & $12.54(4.0)$ & 0.284 \\
\hline
\end{tabular}

Independent samples Student t-test; Results in bold type significant at 5\% level.

the sample had been wearing orthodontic fixed appliances for at least six months. There is an adaptation period of five days after appliance insertion and a decrease in experiences of sensitive teeth, pressure, and pain over time. For patient complaints, no statistically significant changes were observed in retrospective reports within 14 days, three months, and six months after appliance insertion due to patient adaptation. ${ }^{26}$ These findings could support our results for the floor effect. Further research on the B-IFAM is indicated in samples a few days after bonding in order to ascertain whether the high floor effect is sample-specific or a reflection of patient adaptation. Internal consistency was similar to that obtained in the validation process of the original instrument. The original IFAM demonstrated values ranging from 0.56 to 0.89 . The present study found in maintenance impact (MI; $\alpha=0.55$ ) and time constraints (TC; $\alpha=0.55$ ) lower results than the original instrument. The MI result indicates that this sample of Brazilian individuals may not be aware of the negative consequences of appliance breakage in terms of treatment results and the duration of the therapy. Therefore, verbal and written instructions on the care required for the maintenance of a fixed orthodontic device should be provided in subsequent appointments for appliance adjustment. ${ }^{27}$ The TC result may be associated with the difficulty of dealing with both orthodontic dental assistance in the university and school time. However, a study in India demonstrated lower values than the present study for internal consistency in all subscales except for dietary impact, which was the same $(\alpha=0.81) .{ }^{15}$ Therefore, it is suggested that for the Brazilian Portuguese version MI and TC subscales should not be used alone. However, these two subscales should be included in the overall instrument, which showed an excellent Cronbach's alpha coefficient.

The study attained $87.5 \%$ of the sample in test-retest, and the overall score of B-IFAM showed excellent agreement $(\mathrm{ICC}=0.81)$. The manuscript of the original instrument and that from India did not conduct ICC for the overall score of the IFAM. ${ }^{14,15}$ The B-IFAM had better ICC for subscales than the previous studies. No poor agreement was found, while 33\% achieved moderate agreement and $67 \%$ good agreement, although the manuscript of the original instrument found $67 \%$ and $22 \%$ for moderate and good agreement respectively. ${ }^{14}$ In the study from India, just $11 \%$ had moderate agreement, while the other subscales presented poor agreement. ${ }^{15}$ The B-IFAM proved to be stable across time, supporting the reliability of the instrument.

Although previous studies using the IFAM did not calculate construct validity, in this study, $60 \%$ of the coefficients obtained by Pearson's correlation had $\mathrm{p}<0.01$, and $20 \%$ had $\mathrm{p}<0.05$. Therefore, we considered that the Brazilian version of the instrument presented satisfactory construct validity. Likewise, similar results in construct validity were 
achieved in Child-OIDP cross-cultural adaptation and validation in Brazil. ${ }^{28}$

Discriminant validity showed that statistical significances were found in terms of sex regarding the impact on OHRQoL. The mean score achieved by female was higher in the overall score of the B-IFAM and the aesthetics (A) and physical impact (PI) subscales than males. Women perceive oral heath as having a greater impact on quality of life in general than men, whether positive or negative. ${ }^{29}$ Our finding correlates with previous studies where female perceive more impact, ${ }^{30,31,32,33}$ nevertheless, other studies did not find significant differences between sex and impact on OHRQoL. 6 ,7,34

This study has some limitations that should be acknowledged. The first is inherent in the use of a convenience sample. Although several validation studies were based on convenience samples: ${ }^{35,36}$ it has been recognized that a larger sample size could lead to more reliable parameter estimates. ${ }^{37}$ However, a literature review published in 2014 demonstrated that there is no consensus or guidelines on how to calculate sample size for psychometric validation studies. ${ }^{38} \mathrm{~A}$ way to determine a required sample size for this kind of study is by calculating subject to item ratio, with recommendation ranging from 2 to 20 subjects per item. There is also a recommendation for an absolute minimum of 100 to 250 subjects..$^{38}$ As the IFAM was composed by 43 items, and the present study had 161 participants, a ratio of 3.74 was achieved, above the minimal necessary to satisfy the recommendations. Moreover, most participants were from families with a low income. It should be highlighted that the perception of children and adolescents regarding orthodontic outcomes is significantly influenced by socioeconomic factors. ${ }^{39}$ Therefore, this study would have benefited from a more balanced distribution of participants' characteristics.

The B-IFAM has several potential uses in orthodontics. Firstly, it may be used in research to compare the efficacy of different fixed appliance techniques, ${ }^{40}$ such as conventional metallic fixed appliances, esthetic fixed appliances, self-ligating bracket system, and lingual appliances. Secondly, the information provided could be used in orthodontic clinical practice to educate and motivate individuals undergoing orthodontic treatment. ${ }^{14}$ Moreover, it is fundamental in guiding clinicians to understand treatment implications better and improve patient support, resulting in greater patient cooperation. Thirdly, the B-IFAM should prompt the professional to explore the measurement's results to offer high quality services guided by the patient's well-being. Finally, it may provide, along with socioeconomic evaluation, a basis for the efficient allocation of resources in oral health services and evidence to public authorities that treatment should be funded. ${ }^{13}$

The study provides a condition-specific instrument to assess the impact of fixed appliances on the daily lives of Brazilian children and adolescents. However, future evaluations should be carried out with the B-IFAM to assess its psychometric properties fully in population-based studies, allowing the researchers to obtain more accurate estimates. The responsiveness should also be established in order to define the ability of this measure to detect changes in the oral health status of individuals during their orthodontic treatment. ${ }^{41}$ Finally, the performance of longitudinal studies will provide a better understanding of the long-term effects of fixed appliance therapy on individuals' daily lives.

\section{Conclusions}

The B-IFAM overall score and some subscales demonstrated adequate psychometric properties regarding reliability and validity. The results of the present study show that this instrument is feasible for use on Brazilian children and adolescents undergoing orthodontic treatment with fixed appliances.

\section{Acknowledgements}

This study was supported by the National Council for Scientific Development (CNPq), the Coordination for the Improvement of Higher Level Education Personnel (CAPES), and the State of Minas Gerais Research Foundation (FAPEMIG), Brazil. Thank you to the multidisciplinary expert committee: Patricia Alves Drummond de Oliveira, Milene Torres Martins, and Esdras Campos França. 


\section{References}

1. Sischo L, Broder HL. Oral health-related quality of life: what, why, how, and future implications. J Dent Res. 2011 Nov;90(11):1264-70. https://doi.org/10.1177/0022034511399918

2. Gherunpong S, Tsakos G, Sheiham A. Developing and evaluating an oral health-related quality of life index for children; the CHILD-OIDP. Community Dent Health. 2004 Jun;21(2):161-9.

3. Jokovic A, Locker D, Stephens M, Kenny D, Tompson B, Guyatt G. Validity and reliability of a questionnaire for measuring child oral-health-related quality of life. J Dent Res. 2002 Jul;81(7):459-63. https://doi.org/10.1177/154405910208100705

4. Pahel BT, Rozier RG, Slade GD. Parental perceptions of children's oral health: the Early Childhood Oral Health Impact Scale (ECOHIS). Health Qual Life Outcomes. 2007 Jan;5(1):6. https://doi.org/10.1186/1477-7525-5-6

5. Slade GD, Spencer AJ. Development and evaluation of the Oral Health Impact Profile. Community Dent Health. 1994 Mar;11(1):3-11.

6. Feu D, Oliveira BH, Almeida MAO, Kiyak HA, Miguel JA. Oral health-related quality of life and orthodontic treatment seeking. Am J Orthod Dentofacial Orthop. 2010 Aug;138(2):152-9. https://doi.org/10.1016/i.ajodo.2008.09.033

7. Sardenberg F, Martins MT, Bendo CB, Pordeus IA, Paiva SM, Auad SM et al. Malocclusion and oral health-related quality of life in Brazilian school children. Angle Orthod. 2013 Jan;83(1):83-9. https://doi.org/10.2319/010912-20.1

8. Zhang M, McGrath C, Hägg U. Changes in oral health-related quality of life during fixed orthodontic appliance therapy. Am J Orthod Dentofacial Orthop. 2008 Jan;133(1):25-9. https://doi.org/10.1016/i.ajodo.2007.01.024

9. Gameiro GH, Schultz C, Trein MP, Mundstock KS, Weidlich P, Goularte JF. Association among pain, masticatory performance, and proinflammatory cytokines in crevicular fluid during orthodontic treatment. Am J Orthod Dentofacial Orthop. 2015 Dec;148(6):967-73. https://doi.org/10.1016/i.ajodo.2015.05.029

10. Krishnan V. Orthodontic pain: from causes to management: a review. Eur J Orthod. 2007 Apr;29(2):170-9. https://doi.org/10.1093/ejo/cj081

11. Feu D, Miguel JA, Celeste RK, Oliveira BH. Effect of orthodontic treatment on oral health-related quality of life. Angle Orthod. 2013 Sep;83(5):892-8. https://doi.org/10.2319/100412-781.1

12. Anbuselvan GJ, Raja S, Vilvanathan P, Megabob N, Prabhakar $K$. Changing concepts of positive patient communication in dentistry and orthodontics: south Indian perspective. J Pharm Bioallied Sci. 2013 Jun;5(5 Suppl 1):S109-12. https://doi.org/10.4103/0975-7406.113308
13. Cunningham SJ, Hunt NP. Quality of life and its importance in orthodontics. J Orthod. 2001 Jun;28(2):152-8. https://doi.org/10.1093/ortho/28.2.152

14. Mandall NA, Vine S, Hulland R, Worthington HV. The impact of fixed orthodontic appliances on daily life. Community Dent Health. 2006 Jun;23(2):69-74.

15. Nagarajappa R, Ramesh G, Sandesh N, Lingesha RT, Hussain MA. Impact of fixed orthodontic appliances on quality of life among adolescents' in India. J Clin Exp Dent. 2014 Oct;6(4):e389-94. https://doi.org/10.4317/jced.51651

16. Guillemin F, Bombardier C, Beaton D. Cross-cultural adaptation of health-related quality of life measures: literature review and proposed guidelines. J Clin Epidemiol. 1993 Dec;46(12):1417-32. https://doi.org/10.1016/0895-4356(93)90142-N

17. Herdman M, Fox-Rushby J, Badia X. A model of equivalence in the cultural adaptation of HRQoL instruments: the universalist approach. Qual Life Res. 1998 May;7(4):323-35. https://doi.org/10.1023/A:1008846618880PMID:9610216

18. Herdman M, Fox-Rushby J, Badia X. 'Equivalence' and the translation and adaptation of health-related quality of life questionnaires. Qual Life Res. 1997 Apr;6(3):237-47. https://doi.org/10.1023/A:1026410721664

19. Widenfelt BM, Treffers PD, Beurs E, Siebelink BM, Koudijs E. Translation and cross-cultural adaptation of assessment instruments used in psychological research with children and families. Clin Child Fam Psychol Rev. 2005 Jun;8(2):135-47. https://doi.org/10.1007/s10567-005-4752-1

20. Gjersing L, Caplehorn JR, Clausen T. Cross-cultural adaptation of research instruments: language, setting, time and statistical considerations. BMC Med Res Methodol. 2010 Feb;10(1):13. https://doi.org/10.1186/1471-2288-10-13

21. Angle EH. Classification of malocclusion. Dent Cosmos. 1899;41(3):248-64.

22. Terwee $C B$, Bot $S D$, de Boer MR, van der Windt DA, Knol DL, Dekker J et al. Quality criteria were proposed for measurement properties of health status questionnaires. J Clin Epidemiol. 2007 Jan;60(1):34-42. https://doi.org/10.1016/i.jclinepi.2006.03.012

23. Cronbach LJ. Coefficient alpha and the internal structure of tests. Psychometrika. 1951;16(3):297-334. https://doi.org/10.1007/BF02310555

24. Bartko JJ. The intraclass correlation coefficient as a measure of reliability. Psychol Rep. 1966 Aug;19(1):3-11. https://doi.org/10.2466/pr0.1966.19.1.3

25. Cohen J. Statistical power analysis for the behavioral sciences. 2nd ed. Hillsdale: L. Erlbaum; 1988.

26. Sergl HG, Klages $U$, Zentner A. Pain and discomfort during orthodontic treatment: causative factors and effects on compliance. Am J Orthod Dentofacial Orthop. 1998 Dec;114(6):684-91. https://doi.org/10.1016/S0889-5406(98)70201-X 
27. Abreu LG, Lages EM, Abreu MH, Pereira LJ, Paiva SM. Preadolescent's oral health-related quality of life during the first month of fixed orthodontic appliance therapy. J Orthod. 2013 Sep;40(3):218-24. https://doi.org/10.1179/1465313313Y.0000000053

28. Castro RA, Cortes MI, Leão AT, Portela MC, Souza IP, Tsakos $G$ et al. Child-OIDP index in Brazil: cross-cultural adaptation and validation. Health Qual Life Outcomes. 2008 Sep;6(1):68. https://doi.org/10.1186/1477-7525-6-68

29. Mc Grath C, Bedi R. Gender variations in the social impact of oral health. J Ir Dent Assoc. 2000;46(3):87-91.

30. Oliveira CM, Sheiham A. Orthodontic treatment and its impact on oral health-related quality of life in Brazilian adolescents. J Orthod. 2004 Mar;31(1):20-7. https://doi.org/10.1179/146531204225011364

31. Sam G, Seehan S, Al-Shayea M. An analysis of correlation between demand and need for orthodontic treatment among patients in Prince Sattam Bin Abdulaziz University Dental College Clinic, Kingdom of Saudi Arabia. J Int Oral Health. 2015;7(Suppl 2):40-4.

32. Mansor N, Saub R, Othman SA. Changes in the oral health-related quality of life $24 \mathrm{~h}$ following insertion of fixed orthodontic appliances. J Orthod Sci. 2012 Oct;1(4):98-102. https://doi.org/10.4103/2278-0203.105880

33. Bos A, Hoogstraten J, Zentner A. Perceptions of Dutch orthodontic patients and their parents on oral health-related quality of life. Angle Orthod. 2010 Mar;80(2):367-72. https://doi.org/10.2319/031109-141.1

34. Marques LS, Filogônio CA, Filogônio CB, Pereira LJ, Pordeus $I A$, Paiva $S M$ et al. Aesthetic impact of malocclusion in the daily living of Brazilian adolescents. J Orthod. 2009 Sep;36(3):152-9. https://doi.org/10.1179/14653120723139

35. McGrath C, Pang HN, Lo EC, King NM, Hägg U, Samman N. Translation and evaluation of a Chinese version of the Child Oral Health-related Quality of Life measure. Int J Paediatr Dent. 2008 Jul;18(4):267-74. https://doi.org/10.1111/j.1365-263X.2007.00878.x

36. Peker K, Uysal Ö, Bermek G. Cross-cultural adaptation and preliminary validation of the Turkish version of the early childhood oral health impact scale among 5-6-year-old children. Health Qual Life Outcomes. 2011 Dec;9(1):118. https://doi.org/10.1186/1477-7525-9-118

37. Scarpelli AC, Oliveira BH, Tesch FC, Leão AT, Pordeus IA, Paiva SM. Psychometric properties of the Brazilian version of the Early Childhood Oral Health Impact Scale (B-ECOHIS). BMC Oral Health. 2011 Jun;11(1):19. https://doi.org/10.1186/1472-6831-11-19

38. Anthoine E, Moret L, Regnault A, Sébille V, Hardouin JB. Sample size used to validate a scale: a review of publications on newly-developed patient reported outcomes measures. Health Qual Life Outcomes. 2014 Dec;12(1):176. https://doi.org/10.1186/s12955-014-0176-2

39. Joury E, Johal A, Marcenes W. The role of socio-economic position in predicting orthodontic treatment outcome at the end of 1 year of active treatment. Eur J Orthod. 2011 Jun;33(3):263-9. https://doi.org/10.1093/ejo/cjq080

40. Cunningham SJ, Garratt AM, Hunt NP. Development of a condition-specific quality of life measure for patients with dentofacial deformity: I. Reliability of the instrument. Community Dent Oral Epidemiol. 2000 Jun;28(3):195-201. https://doi.org/10.1034/i.1600-0528.2000.280305.x

41. Abreu LG, Melgaço CA, Lages EM, Abreu MH, Paiva SM. Effect of year one orthodontic treatment on the quality of life of adolescents, assessed by the short form of the Child Perceptions Questionnaire. Eur Arch Paediatr Dent. 2014 Dec;15(6):435-41. https://doi.org/10.1007/s40368-014-0135-y 